8.00) for RSV-negative compared to 9.76 days, 95\%C. I. (8.3111.21) for other non-RSV. The $\mathrm{p}$ value was 0.723 when comparing the length of stay in infants who tested positive RSV to those tested negative RSV, while the p-value was (0.059)when comparing RSV-positive to other non-RSV viruses. There was a statistically significance difference in length of stay for RSV negative compared to other non-RSV viruses $(p=0.010)$.

Conclusions Our data showed, there is no difference in length of stay in infants hospitalised with RSV-positive bronchiolitis compared to the group with RSV-negative; however the length of stay was statistically significant longer with Non-RSV viruses compared to RSV-negative infection.

\section{PO-0012 MANAGING CHILDREN WITH PROTRACTED BACTERIAL BRONCHITIS}

H Abdelrhim, S Paul. Neonates, North Bristol NHS Trust, Bristol, UK

10.1136/archdischild-2014-307384.692

Introduction It is important that health professionals consider a list of differential diagnoses when faced with a child with chronic cough (acute cough usually lasts less than three weeks). Potential causes include: asthma, cystic fibrosis, foreign body aspiration, anatomical abnormalities of the airways and other disorders.

Studies have shown that children with chronic wet cough often have bronchitis and this is evident on bronchoscopy. Protracted bacterial bronchitis (PBB) is defined as persistence of isolated wet cough lasting more than four weeks and responding to antibiotic treatment.

Diagnosis Clinical is achieved by presence of wet cough lasting $>4$ weeks. It is primarily a neutrophilic disease and presence of respiratory bacterial pathogens has been demonstrated in bronchoalveolar lavage. Prolonged course of oral antibiotics of 2-3 weeks is suggested as the initial therapy. Treatment is likely to result in symptom resolution and may minimise the risk of developing bronchiectasis. Chest X-ray may be performed in some cases and will be found to be normal in most instances. Sputum culture may also be sent in older children.

Diagnosis may be confirmed by bronchoscopy with BAL followed by bacterial study; however, such an invasive approach may not be deemed necessary or may not be readily available in most cases.

Treatment The British Thoracic Society (BTS) advises using four to six weeks of oral antibiotics. 4 In the absence of a bacterial culture a prolonged course of either amoxicillin or a macrolide antibiotic is suggested in most children with PBB.

\section{PO-0013 THERAPEUTIC PRACTICE OF ACUTE RESPIRATORY INFECTIONS IN YOUNG CHILDREN}

A Holban, A Rotari, E Vlad, O Turcu. Pediatrics, State Medical and Pharmaceutical University Nicolae Testemitanu, Chisinau, Moldova

\subsection{6/archdischild-2014-307384.693}

Background In Moldova, acute respiratory infections (ARI) constitute $2 / 3$ of infant morbidity and are in the top causes of mortality in last 20 years.

The aim of the study was to analyse the practical aspects of ARI treatment in young children in the light of national and international guidelines.
Materials and methods It was performed a retrospective study of 100 medical records of inpatients with IRA hospitalised in 2012. Mean age of patients was $12.58 \pm 1.09$ months, including $66 \%$ of infants. Analysis included: clinical signs of disease onset, clinical outcome and cause of hospitalisation, laboratory tests, home and in hospital treatment, treatment compliance with guidelines.

Results The most common syndrome at admission was fever (36\% patients), but antipyretics were administered at lower levels than considered febrile, contrary to international guidelines.

In 93\% cases were given anti-viral drugs, mucolytics, topical treatment, in $45 \%$ cases - antibacterial treatment. The antibiotics used at home were not according to local protocols in $31 \%$ cases. The average length of home treatment was $4.6 \pm 0.3$ days.

Serum levels of leukocytes $\left(8.9 \pm 0.35 \times 10^{9} / \mathrm{l}\right)$ at admission were not suggestive of bacterial aetiology.

Antibacterial treatment was applied in all patients. Antibiotic was changed within the first $24 \mathrm{~h}$ in $35.3 \%$ of children, after 48 $\mathrm{h}$ of hospitalisation - in $14.7 \%$ children, and after $72 \mathrm{~h}$ or more - in 50\% children. Unjustified polypharmacy was found in 51\% cases.

Conclusion Antibacterial treatment of ARI is not always according national and international protocols, but there are divergences between national and international guidelines.

\section{PO-0014 OTITIS MEDIA IN CHILDREN WITH RECURRENT SOMATIC PATHOLOGY}

${ }^{1} \mathrm{~S}$ Diacova, ${ }^{2} \mathrm{~N}$ Revenco, ${ }^{3} \mathrm{~T}$ Culesin, ${ }^{4} \mathrm{~L}$ Cerempei, ${ }^{5} \mathrm{O}$ Diacova, ${ }^{6} \mathrm{~V}$ Desvignes. "Otorhinolaryngology, State University of Medicine and Pharmacy "Nicolae Testemitanu", Chisinau, Moldova; 'Paediatrics, State University of Medicine and Pharmacy "Nicolae Testemitanu", Chisinau, Moldova; ${ }^{3}$ Allergology, Institute for Maternal and Child Healthcare, Chisinau, Moldova; " Pediatrics Gastrology, State University of Medicine and Pharmacy "N. Testemitanu", Chisinau, Moldova; ${ }^{5}$ Student, State University of Medicine and Pharmacy "N. Testemitanu", Chisinau, Moldova; ${ }^{6}$ Pediatrics, Society "Pédiatres Du Monde", Nantes, France

\subsection{6/archdischild-2014-307384.694}

Background and aims Subclinical evolution of otitis media $(\mathrm{OM})$ in childhood predetermines late diagnostics and treatment, chronicity and complications. We compared incidence and clinic evolution of otitis media in children with recurrent somatic pathology and healthy children.

Methods We monitored middle ear status of children at the age between 1 and 7 years with recurrent respiratory pathology (Group R), recurrent gastrointestinal pathology (Group G), and healthy children (Group $\mathrm{H}$ ) by tympanometry and otoscopy during 1 year. Complete audiological assessment and otomicroscopy were carried out in children who failed the screening tests during 3 months. Treatment approaches included medical and surgical methods and treatment of somatic pathology. Surgical findings in different groups were compared.

Results There were significant dependence of OM development and evolution from somatic pathology and age of children. OM chronicity rate was $35 \%$ in group R, $16 \%$ in group $\mathrm{G}$ and $4 \%$ in group $\mathrm{H}$. Chronic and recurrent forms of OM correlated to respiratory tract infection-prone children, aged younger than 5 years of life. Chronic inflammatory changes of tympanic cavity were most evident in Group G.

Conclusions High rate of OM chronicity was predetermined by somatic pathology. These groups of patients needed comprehensive diagnostics and intensive treatment, including the surgical 
one. In healthy children $\mathrm{OM}$ was a relatively rare, temporary and benign condition. Middle ear monitoring is important for children with recurrent somatic pathology.

\section{PO-0015 EVALUATION OF PEDITRIC PATIENTS PRESENTING IN ITP IN KING ABDULAZIZ UNIVERSITY HOSPITAL}

N Fida, S Alshareif. Pediatric, King Abdul Aziz University-Faculty of Medicine, Jeddah, Saudi Arabia

10.1136/archdischild-2014-307384.695

Background Immune thrombocytopenia (ITP) is an acute disease of short duration with mild symptoms and with estimated incidence in children is approximately 1.9 to 6.4 cases per 100,000 per year. The management of acute ITP of childhood has generated controversy for many years. Although most children can be managed by careful monitoring, a small proportion of children will suffer from bleeding. ${ }^{1}$

The Aim The aim of our study is to characterise bleeding severity, platelets count, the management over the past 2 years.

Material and methods All patients diagnosed with ITP, with a first visit to King Abdelaziz University Hospital Jeddah, Saudi Arabia during last2 years will eligible for this study. Demographic, laboratory, and treatment data were collected through a questionnaire.

Result The mean age of the patients was 6.5 years (range, 1 month to 16 years), 18 boys and 14 girls. The mean plateletcount at presentation was $22.3 \times 109 / \mathrm{L}$ (range, 1 to $108 \times 109 /$ L). Bleeding symptoms were found in 22 cases (68.80\%). 19 (59.40\%) had skin bleeding, 15 (46.9\%)had mucosa bleeding, and $4(12.50 \%)$ had organs bleeding. Bone marrow aspiration and laboratory tests (antinuclear antibodies, human immunodeficiency and hepatitis C virus) were performed for18 (56.30\%) children.26 (81.1\%) of patients received Immunoglobulin, and 9 $(28.10 \%)$ received corticosteroids.

Conclusion Most children with ITP treated by paediatrician received Immunoglobulin, we recommend that physician should follow the new guideline tools on ITP diagnosis and management to reduce the number of children requiring intervention and possible drug-induced side effects.

\section{PO-0016 HENOCH SCHOLEIN PURPURA IN CHILDREN: ABOUT 24 CASES}

I Maaloul, L Sfaihi, J Telmoudi, Y Bel Fitouri, H Aloulou, TH Kamoun, M Hachicha. Pediatric Department, CHU Hedi Chaker Sfax Tunisia, Sfax, Tunisia

\subsection{6/archdischild-2014-307384.696}

Background Henoch Scholein Purpura is the most frequent vasculitis in children. Its diagnosis is clinical and prognosis is often favourable.

Aim To precise the epidemiological, clinical, biological profiles and course of the disease.

Methods Retrospective study over 10 years (January 2005-January 2014) of 24 children with Henoch-schonlein vasculitis. The diagnosis was based on the association of non-trombocytopenic purpura, arthritis or arthralgia and abdominal pain.

Results The mean age of patients was 5,2 years with male predominance (sex-ratio: 1.8). Purpura was observed in all cases. Articular manifestations were noted in 16 cases $(69,5 \%)$. Digestive manifestations were noted in 11 cases; it involves acute gastro-intestinal bleeding (4 cases), abdominal pain (6 cases) and diarrhoea (1 case). Upper digestive gastrointestinal endoscopy was done in 3 cases; it showed erosive gastritis in one case. Ultrasonography showed intussusception in 2 patients. Moderate renal manifestations (microscopic hematuria, moderate proteinuria) were observed in 5 children. Five patients required corticosteroid therapy. The evolution was favourable in all cases.

Conclusion The Henoch-Sconlein purpura is generally a benign disease. prognosis depends on the risk digestive complications at the acute phase and renal damage at long-term.

\section{PO-0017 ETIOLOGICAL DIAGNOSTICS OF THE BOY'S FUNCTIONAL RETENTION OF SEXUAL DEVELOPMENT}

VA Popova, AA Kozhin, MA Daurbekova, AA Afonin. Pediatric Department, Rostov Scientific-Research Institute of Obstetrics and Pediatrics, Rostov-on-Don, Russia

\subsection{6/archdischild-2014-307384.697}

Early diagnostics of pathology of ecological genesis is poorly developed. In this connection the goal of this scientific work was determination of elements composition of urine by spectral method and study of concentrations proportion of chemical elements (ChE) in the boys with functional retention of sexual development (FRSD) in comparison with morphobiochemical indices of the reproductive system. It was shown that in boys of 12-13 with FRSD on the background of alimentary obesity (1 group) the level of leptin in blood was two times as much than a control level and the indeces of gonadotropins, and testosterone were $30 \%$ less than in children of a comparison group. At the same time ChE concentrations and their proportions didn't reliably differ from the control indices.

In the boys of the 2- nd group (with deficiency of body mass) leptin indices were insignificantly less than in control indices, but the level of sexual hormones was lower than a norm more than in 3 times. The indice of selenium was 2, 5 times lower than in the boys of the 1-st group and the comparison contingent, but the proportion index of zinc/lead concentrations did not differ from the control index. The level of lead in urine of all teenagers under examination was approximately equal.

Thus our data show that aetiology of FRSD in the children was different (hormonal and microelements character) and this fact causes different type of the therapy.

\section{PO-0018 PLASMA HOMOCYSTEINE IN OVERWEIGHT AND OBESE SCHOOL CHILDREN}

${ }^{1} \mathrm{~L}$ Dimitriu, ${ }^{2} \mathrm{AG}$ Dimitriu. ${ }^{1}$ Pediatric Cardiology, Medex Medical Center, lasi, Romania, ${ }^{2}$ Pediatric Cardiology, University of Medicine and Pharmacy, lasi, Romania

\subsection{6/archdischild-2014-307384.698}

High values of plasma homocysteine (Hcy) level were highlighted and in the child obesity, elevated levels can be an independent risk factor for cardiovascular disease. Objective. Research of plasma Hcy values in overweight and obese school children compared with a control group of non obese children and without cardiovascular diseases and correlations with blood pressure values and blood levels of total cholesterol and tryglycerides. Methods. The authors researced in ambulatory 36 children (8-18 years) of which 26 overweight and obese and 10 non-obese healthy children, without cardiovascular diseases. All children were investigated by clinical exam, body mass index, determination of plasma levels of homocysteine in the morning after 12-hour fasting, total cholesterol, tryglyceride, bloog 